\title{
An adaptive speech signal processing for COVID-19 detection using deep learning approach
}

\author{
Kawther A. Al-Dhlan ${ }^{1}$
}

Received: 9 December 2020 / Accepted: 29 July 2021 / Published online: 21 August 2021

(c) The Author(s), under exclusive licence to Springer Science+Business Media, LLC, part of Springer Nature 2021

\begin{abstract}
Researchers and scientists have been conducting plenty of research on COVID-19 mo its outb eak. Healthcare professionals, laboratory technicians, and front-line workers like sanitary workers, data collectors $n$ putting tremendous efforts to avoid the prevalence of the COVID-19 pandemic. Currently, the reverse tr nscr ption polymerase chain reaction (RTPCR) testing strategy determines the COVID-19 virus. This RT-PCR processing Alum xpensive and induces violation of social distancing rules, and time-consuming. Therefore, this research we ${ }^{-1}$ introduc generative adversarial network deep learning for quickly detect COVID-19 from speech signals. This propos d $\mathrm{d}$ consists of two stages, pre-processing and classification. This work uses the least mean square (LMS) filter algor thm to remove the noise or artifacts from input speech signals. After removing the noise, the proposed generative mersarial etwork classification method analyses the melfrequency cepstral coefficients features and classifies the CO TD-19. ynals and non-COVID-19 signals. The results show a more prominent correlation of MFCCs with various COVD-1, ough and breathing sounds, while the sound is more robust between COVID-19 and non-COVID-19 models. As smp red $\mathrm{w}$ the existing Artificial Neural Network, Convolutional Neural Network, and Recurrent Neural Network, the p. osed JAN method obtains the best result. The precision, recall, accuracy, and F-measure of the proposed GAN $96.54 \%, 0.15 \%, 98.56 \%$, and 0.96 , respectively
\end{abstract}

Keywords COVID-19 - Automatic speecr recognition, Generative adversarial network - Mel-frequency cepstral coefficients

\section{Introduction}

COVID 19 is a respirat severe respiratory d'sease, vid 2 (SARS-CoV-2) (Trouvain \& Truong, 01 . Many worldwide have an infection rate between and 10\% nany countries, and the condition has ne heer off cially reported (James, 2015). Figure 1 shows the Ev "utio of COVID-19 cases and deaths up to aug st 2 20. $T_{1}$ development direction began on January a serious atrol estimates across country lockdowns and scaling-up of the confinement offices in emergency clinics (Sakai, 2015; Schuller et al., 2014). Lockdown process is valuable because it gives excellent time and scope of testing for a maximum number of patients. Reverse transcription

Kawther A. Al-Dhlan

K_Aldhlan@Hotmail.Com

1 Information and Computer Science Department, University of Ha'il, Hail, Kingdom of Saudi Arabia polymerase chain reaction (RT-PCR) is one of the best methods for analyzing and detecting COVID 19 within $48 \mathrm{~h}$ (Ghosh et al., 2015, 2016a, 2016b; Usman, 2017).

The testing interaction incorporates (i) avoid social distance, it grows the chances for effectively spreading the infection, (ii) the expense of having chemical reagents and widgets, (iii) testing time is high, and (iv) obstacles in hugescale spread. Attempts to predict a more significant number of COVID-19 cases have led to productive recommendations on innovative solutions for medical services (Botha et al., 2018; McKeown et al., 2012; Porter et al., 2019; Windmon et al., 2018). In particular, progress needs to be made to test simpler, less expensive, and more accurate diagnosis approaches (Breathing sounds for COVID-19, 2020; Indian Institute of Science, 2020; Menni et al., 2020). A few countries have changed the essential, policymaking, and economic restructuring of medical services. The attention is also focused on the purpose of diagnosis tools, innovation arrangements that can be facilitated quickly for pre-screening, and exploring less expensive options than RT-PCR 
Fig. 1 Ratio of COVID-19 cases up to August 2020. Source https://arxiv.org/pdf/2005. 10548.pdf

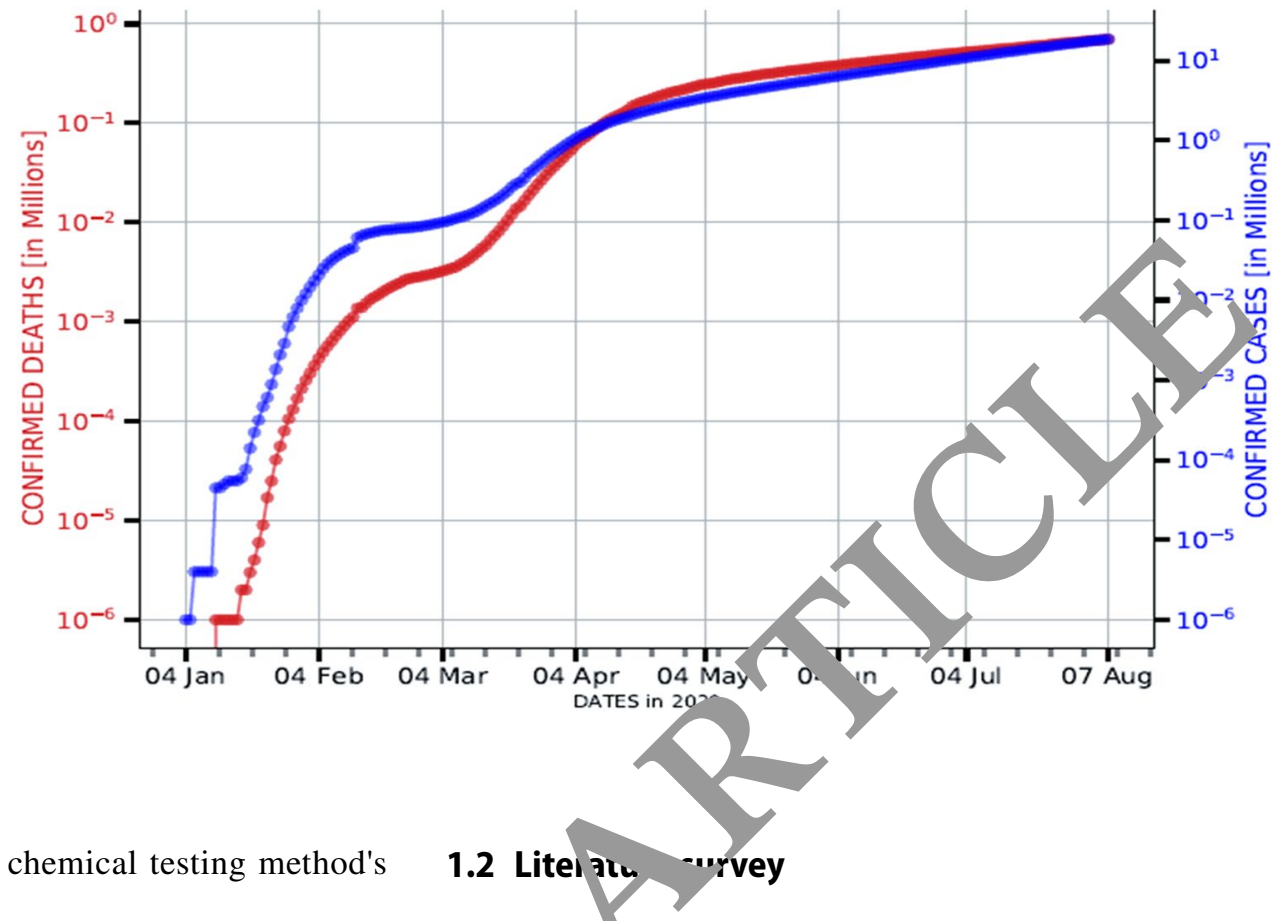

test, which will overcome the chemical testing method's drawbacks.

COVID 19 identification and testing development are being carried out in various laboratories around the world. The WHO and the CDC have identified speech loss as one of the main symptoms of this infectious illness, pre ent ing as difficult coughing, a dry cough, and chest nan. $\mathrm{c}$ to 14 days after exposure to the virus. Clinical sting pro jects that incorporate structural and physiol gica 'Huber \& Stathopoulos, 2015) improvements in ale unpredi able respiratory system are speech breathin models. Based on our observations, we believe that speech s. als $\mathrm{r}$. ght blame the shift in COVID 19 detection.

Bringing together an enormous alc set of breathing sounds and respiratory dis skil from clinical experts

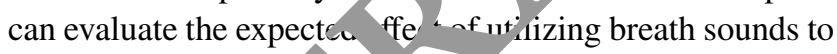
recognize COVID-1 Indica ons using deep learning methods (Thorpe et $\mathrm{a}^{1}, 2$ 1). This work's primary purpose is to supplement ex ting ch rical testing methods by replacing them with ow ast fas process, and high accuracy. This research wor rovi es efforts in this direction.

1.1

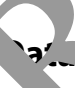

First, to generate data on healthy and unhealthy sound samples, including COVID-19 identification. The generated samples are analyzed using the proposed generative adversarial network method. It has built on assistive mathematical models that identify biomarkers from sound models. Progress should be made when creating task data at this stage.
C-aral stuc ies have proposed sound features that detect sym $_{\mathrm{f}} \mathrm{jms}$ and vocal signals in respiratory diseases in scen years.

is the examination has focused on expanded COVID 9, ongoing works have started researching the utilization of deep neural networks by people to characterize sick dependent on cough sounds. Venkata Srikanth and Strik (2019) use Convolutional Neural Network (CNN) and Recurrent Neural Network (RNN) architectures for breath occasion discovery as a likely pointer of COVID19 recognition. As of late, Basheer et al. (2020) used the CNN architecture to perform direct COVID-19 symptomatic groupings dependent on cough sounds. The work in Chon et al. (2012) uses a learning step technique of deep finding out how to do a similar analysis to our own, with an F1 score of 0.929 , which is not at all like the methods discussed in this article.

More recently, microphones in devices, for example, cell phones and wearable devices, have been abused for voice examination. In Rachuri et al. (2010), the microphone audio is utilized to comprehend the client's current circumstance. This data is assembled to briefly look at the environmental factors in places around the city alone. In COVID-19 recognition (Nandakumar et al., 2015), a sensor recognizes clients' feelings through the telephone's receiver wild Gaussian compound models. In Oletic and Bilas (2016), Pramono et al. (2017), Praveen Sundar et al. (2020), the authors distinguished COVID-19 in the investigation using sound samples based on different machine learning methods. 


\section{Proposed COVID-19 detection using speech signal}

The generative adversarial network with speech signalbased COVID-19 detection system is shown in Fig. 2. The proposed system consists of two stages, pre-processing and classification. The Least Mean Square filter removes the artifacts or noise from the input speech signal in the pre-processing step. After completing the pre-processing process, the GAN classifier analyses the filtering signal to classify COVID-19 and non-COVID-19 signals.

\subsection{Noise reduction using LMS}

Typically, all biomedical signals contain noise or artifacts. Hence, before classifying the signals, we need to remove the noise or artifacts for accurate results. In this research work, the Least-Mean-Square (LMS) filtering method is used to remove the noise. As compared with other filters, the LMS decreases the variance of weights to stabilize the signal using the Lagrangian approach. This Lagrangian method has a nonlinear transformation rule, and it differentiates the input and output derivatives, which solves the optimization problem of the LMS algorithm. The LMS pre-processing steps are discussed below.

\subsubsection{LMS algorithm}

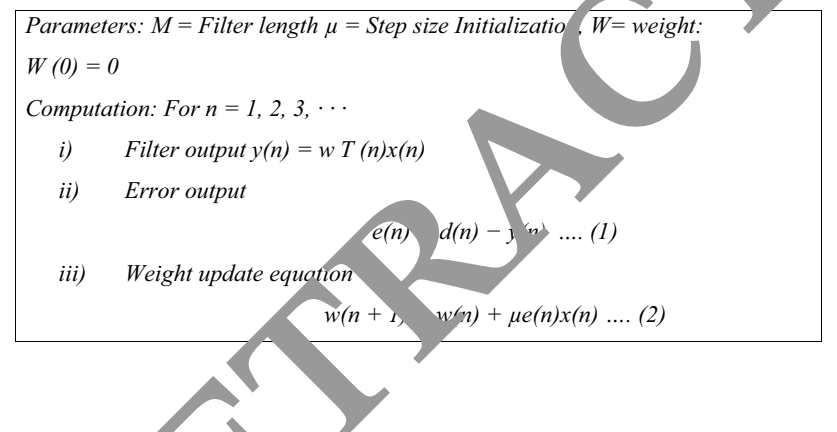

The optip atic.. is ues is overcome using the strategy of Lagranoe mu vlie s. The equation of Lagrangian is given in $F$ r. (3
$\mathrm{L}(\mathrm{w}(\mathrm{n}+1))=\|\S \mathrm{w}(\mathrm{n}+1)\|^{2}+\operatorname{Re}\left[\lambda * \mathrm{e}^{[\mathrm{n}+1]}(\mathrm{n})\right]$

where $\mathrm{w}(\mathrm{n}+1)=$ tap weight vector, $\S \mathrm{w}(\mathrm{n}+1)=\mathrm{w}(\mathrm{n}+1)$ $-\mathrm{w}(\mathrm{n})$ in the tap-weight vector $\mathrm{w}(\mathrm{n}+1)$ with respect to its old worth $\mathrm{w}(\mathrm{n})$.

Here $\lambda^{*}$ is known as the Lagrange multiplier in this way getting the famous variation rule in 3, with the standardized advance size gave by $\mu=\hat{\mu} / \| \lambda$. T e last restriction is unnecessarily obstructive in ope plications; therefore, an additional inter ing sc lution is derived when we relax it.

\subsection{GAN classifier}

This section discusse the 'nerative Adversarial Network method's workin o nction b sed on COVID-19 detection from the spec $\mathrm{si}$ The optimal threshold value of COVID-19 is abo $1.2 \mathrm{~Hz}$, and non-COVID-19 is below $0.60 \mathrm{~Hz}$. invest gation model's unsupervised learning piece is dive $v^{d}$ for the Deep Convolution Generative Adversaria Network (GAN) design or DCGAN.DCGAN COn ins two main blocks known as generators and discrimi ators, and these blocks are trained using min-max a ar gement. The Generator receives the samples from raidom distributions variance of output conditions. The discriminator takes samples from either the output of the generator or actual speech samples from the dataset. During training, the discriminator utilizes the cross-entropy loss function to distinguish the number of classified models completely in genuine models, and the Generator classifies the number of good ones. The mathematical calculation of real $(y)$ and predicted $(\hat{y})$ values are defined in Eq. (4).

$L(w)=-\frac{1}{N} \sum_{n=1}^{N}\left[y_{n} \log \hat{y}_{n}+\left(1-y_{n}\right) \log \left(1-\hat{y}_{n}\right)\right]$

where $w=$ weights of learned vectors, $N=$ size of samples.

For this calculation, 1 represents the real sample, and 0 represents the generated samples. The prediction of discriminator $\left(\hat{y}_{r}\right)$ is computed using Eq. (5).
Fig. 2 Bleck diagram of COVID-19 detection

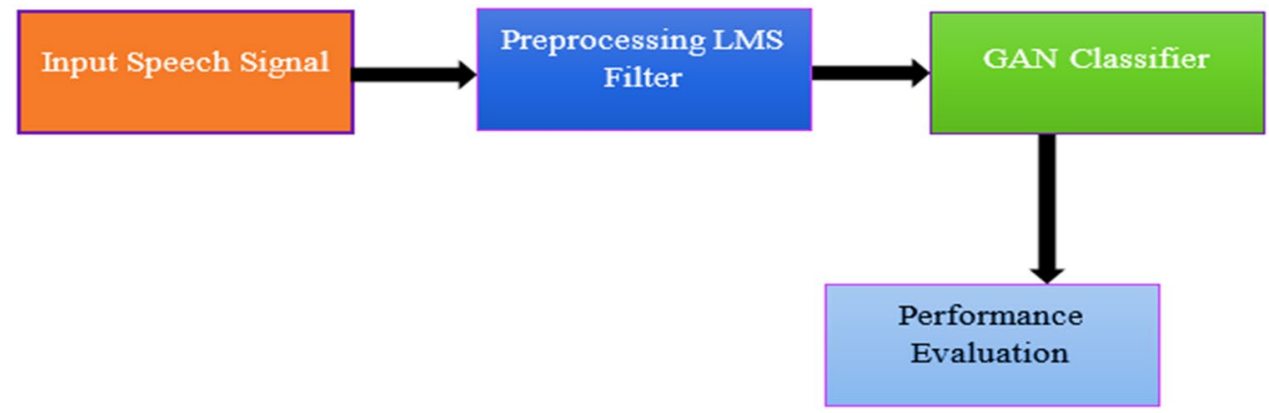


$L_{r}(W)=-\frac{1}{N} \sum_{n=1}^{N} \log \hat{y}_{r}, n$

All the correct predictions are considered as zero for this case. Similarly, the $\hat{y}_{g}$ discrimination represents prediction. Therefore, the correct prediction of the cross-entropy function is simplified by using Eq. (6)

$L_{f}(W)=-\frac{1}{N} \sum_{n=1}^{N} 1-\log \hat{y}_{g}, n$

The generator also uses cross-entropy loss, which should be interpreted in terms of fallen generator outputs into the real sample. The cross-entropy loss of the Generator is computed using Eq. (7).

$L_{g}(W)=-\frac{1}{N} \sum_{n=1}^{N} \log \hat{y}_{g}, n$

If the generator has low loss, the proposed system gives the discriminator results as accurate.

This process leads the Generator to produce output and looks like an actual sample of well-trained iterations shown in Fig. 3. Both the activation of the valence classifier crossentropy misfortune function to reduce the loss. The cresu entropy function is discussed by Eq. (7): the valence acti-

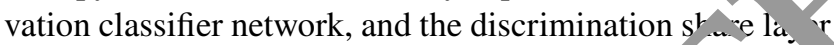
model, which learns the characteristics. The volution filter is effectively used for the valence class ficat task to activate the classification network to d stinguish be ween actual and generated speech samples.
Figure 4 discusses the overall process for describing the proposed Deep Convolution Generative Adversarial Network with record cough-breath sound, extract audio features, split the training/testing ratio, and performance validation. The testing and training ratio is 80:20. The classification response of the proposed COVID-19 detection system's performance is validated using precision, recall, and accuracy. $C$ mpared to other deep learning methods, GAN does not requ labe d data; they can be trained using unlabeled data to $\mathrm{h} r \mathrm{r}$ the data's internal representations. So the perfor ance is unomatically improved.

Precision It is the fraction of elevant spcech samples among the retrieved speech s- nplc The lathematical formula of precision is shown $\eta \mathrm{E}_{\mathrm{t}}$ (8).

Precision $(P)=\frac{T_{i}}{T_{P} F_{p}}$

Recall It is the ction of retrieved relevant speech samples among al olevant sp ch samples. The mathematical formula of recall is shy Eq. (9).

$$
(\mathrm{R})=\frac{T_{p}}{T_{p}+F_{n}}
$$

curacy Accuracy is the ratio of correctly classify the COVID-19 samples from the total number of samples. The following Eq. (10) is used to compute the accuracy.

$$
\text { Accuracy }=\frac{T_{p}+T_{n}}{\left(T_{p}+T_{n}+F_{p}+F_{n}\right)}
$$

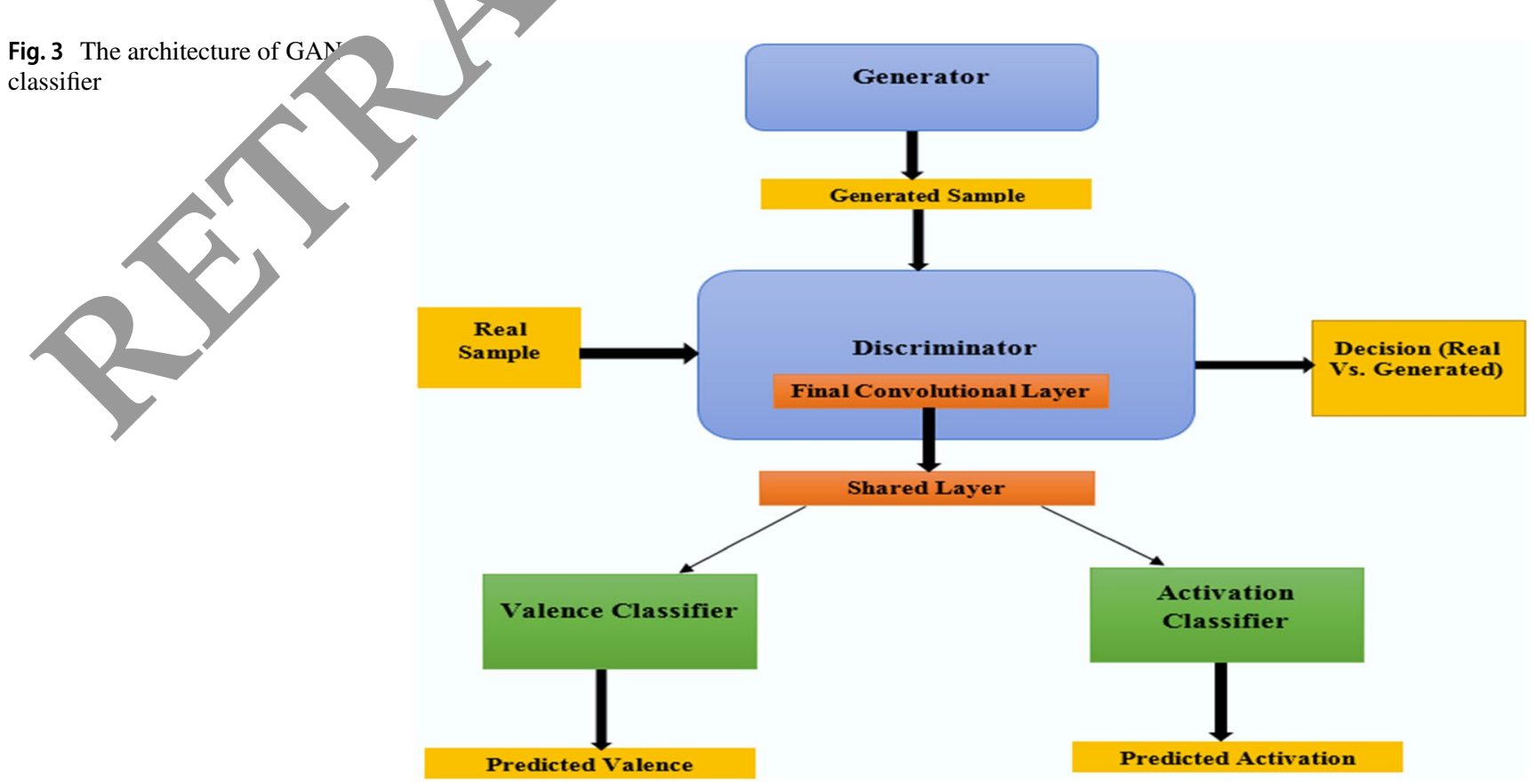



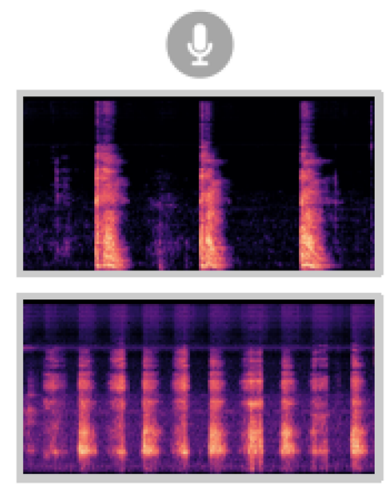

Record Cough
and Breathing
Sounds
Extract Audio Features and

(2) combine them with the output of a pre-trained audio neural network
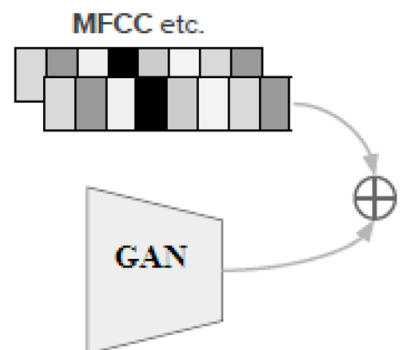

ut 3

inot 80/20 Train and Test sets

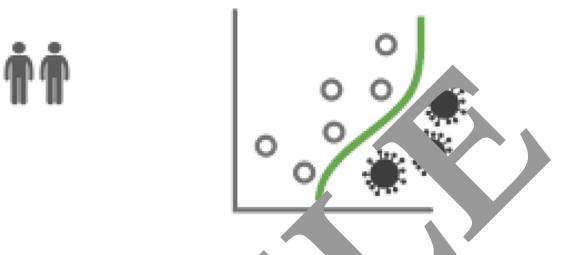

Train Nodel to Detect

ovid 9 Sounds and

vanuate the accuracy

Fig. 4 Overall process of proposed method

where $T_{p}=$ true positive, $T_{n}=$ true negative, $F_{p}=$ false positive, $F_{n}=$ false negative.

\section{Simulation results and discussion}

Simulation results and performance analysis of the $\mathrm{p}_{\text {. }}$ posed COVID 19 detection system are disc $s$ s in this section. This work aims to classify speect samplo from normal and abnormal people, inclu e to identi ying COVID-19 patients.

The input speech signal of the propo COVID-19 detection is depicted in Fig. 5. The int inal's frequency range is $8 \mathrm{kHz}$.

Fig. 5 Noisy signal

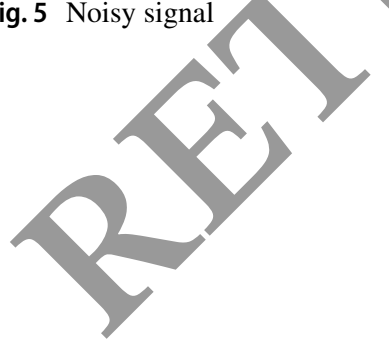

Time- nin representation of proposed Generative Adversaric 1 Neu Network-based COVID-19 detection is shown in Fi, 6.

proposed Generative Adversarial Neural Networkbased ime-domain representation of the noise signal of YVD-19 detection is shown in Fig. 7.

The proposed Generative Adversarial Neural Networkbased time and frequency response of the filtered signal COVID-19 detection is shown in Fig. 8.

Figure 9 shows the Spectrogram of the pre-processed speech signal. The Spectrogram splits the Window that allows overlapping elements in each section with windows notation.

Figure 10 shows the simulation results of validation accuracy and loss in training. The proposed COVID-19 detection

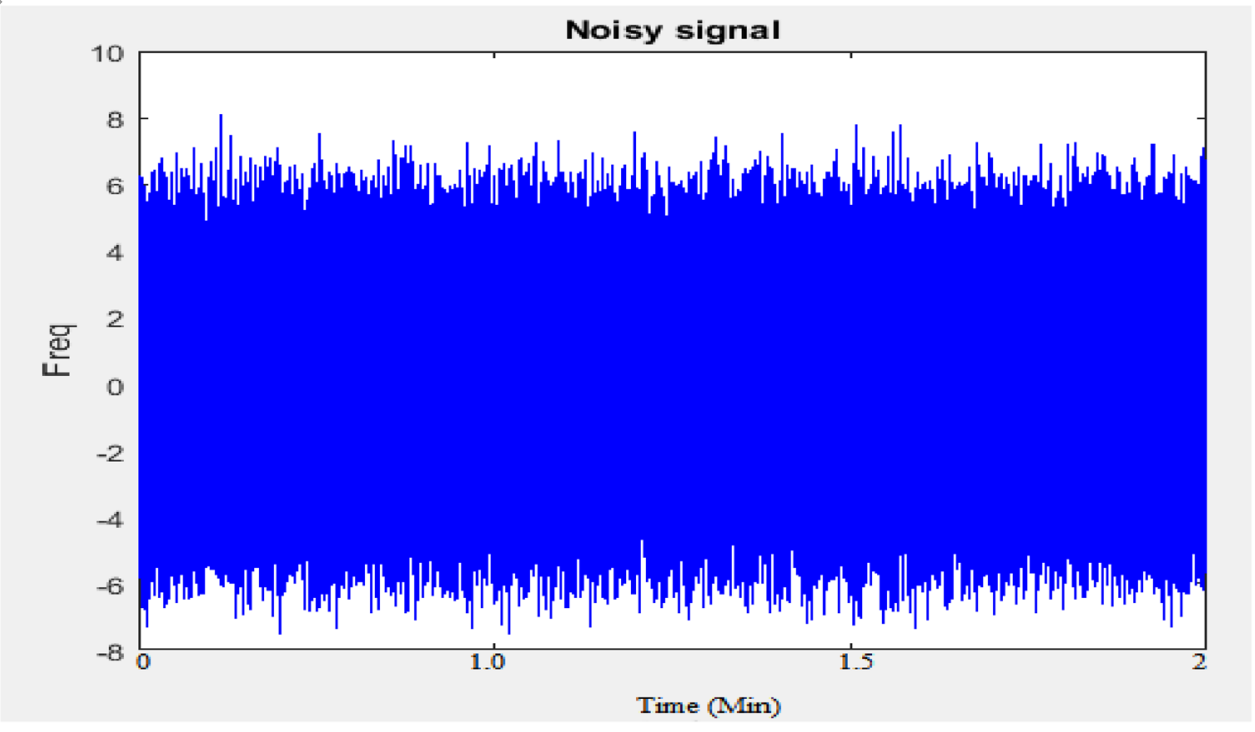


Fig. 6 Time domain representation of the desired signal

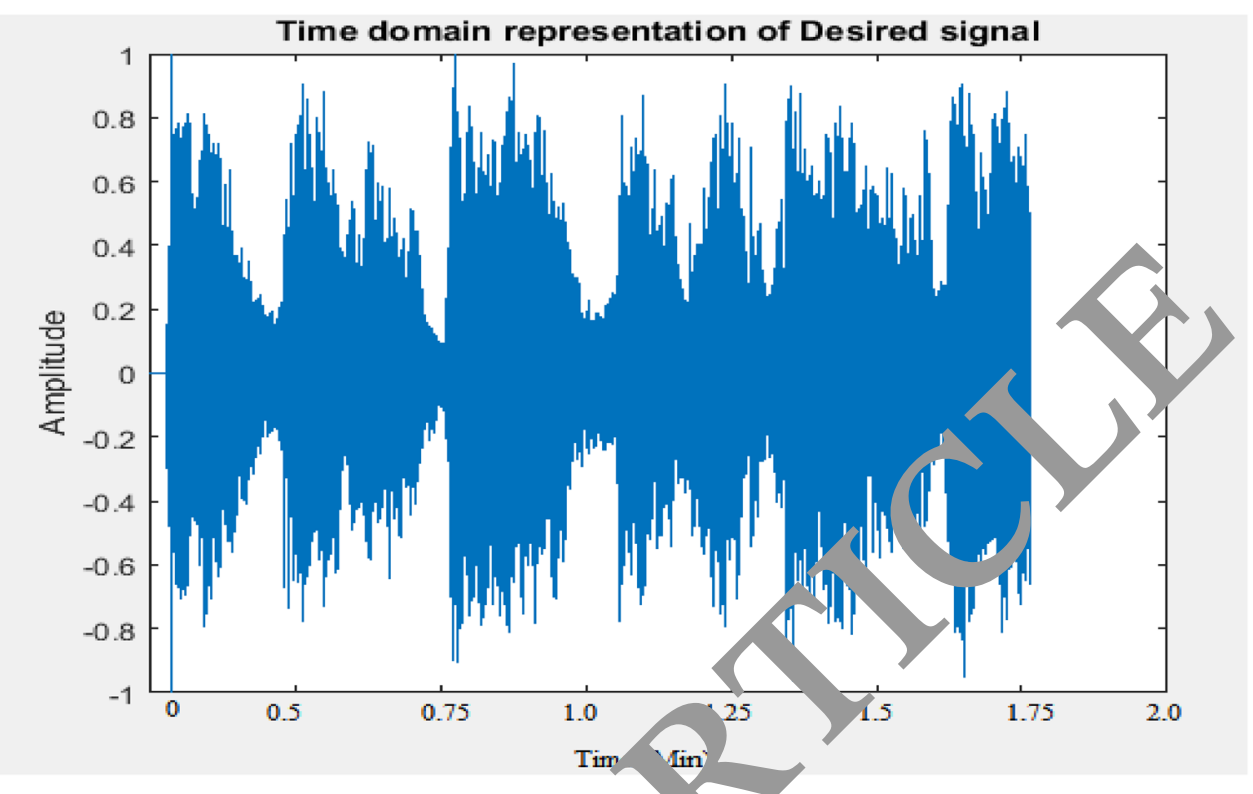

Fig. 7 Time domain representation of noise signal

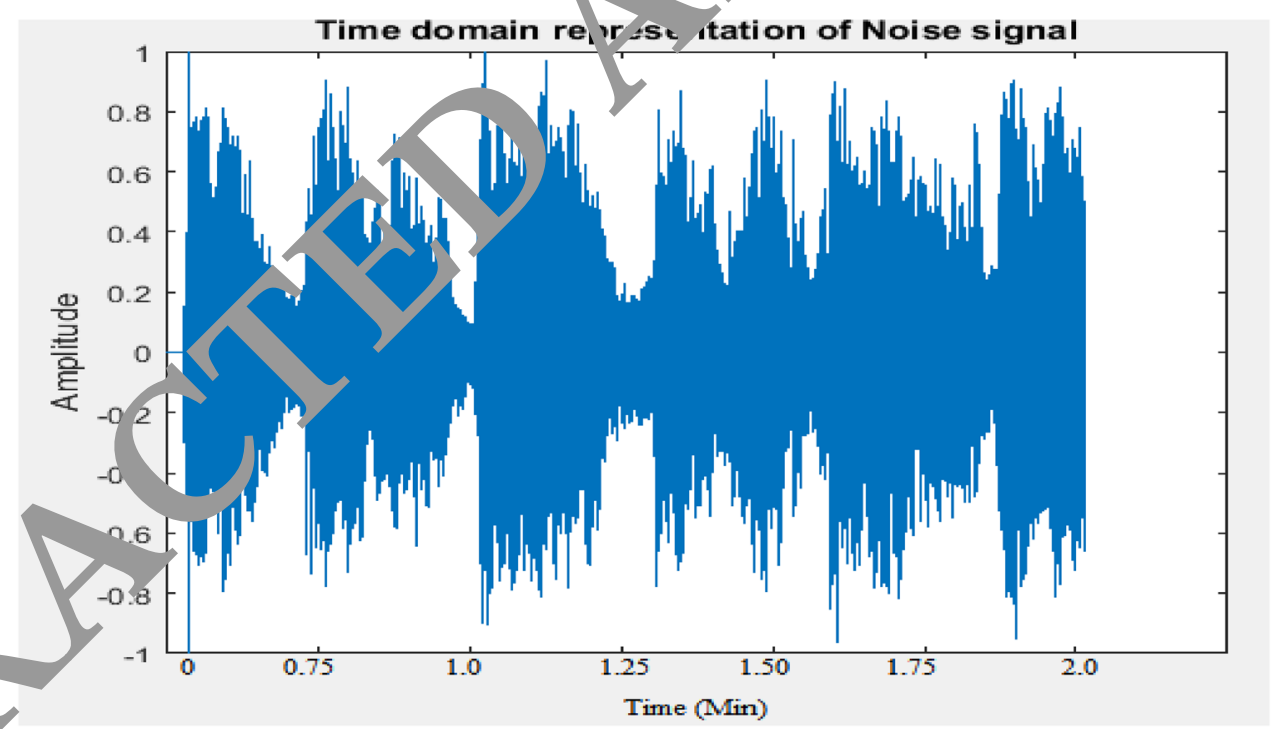

system redur es the valida on loss and increases the validation accuracy, $m_{\mathrm{t}}$ ir $g$ the model learning low mean squared error.

Fig 11 an $T$.ole 1 discuss the performance analysis of the rop sed CCVID-19 classification system with existing metho As compared with existing methods, the proposed GAN me nod achieves a good result. The precision, recall, accuracy and F-measure are 96.54\%, 96.15\%, 98.56\% and $0.96 \%$ respectively.

\section{Conclusion}

This research work introduces Generative Adversarial Network for the detection of COVID-19 symptoms from a speech signal. Typically, speech signals contain intrinsic information regarding the physiological as well as emotional conditions of humans. Accurate measurement of such physiological parameters using speech signals has facilitated real-time, remote monitoring of infected/ symptomatic individuals and early detection of COVID19 symptoms, resulting in containing the spread of the 
Fig. 8 Time and frequency response of a filtered signal
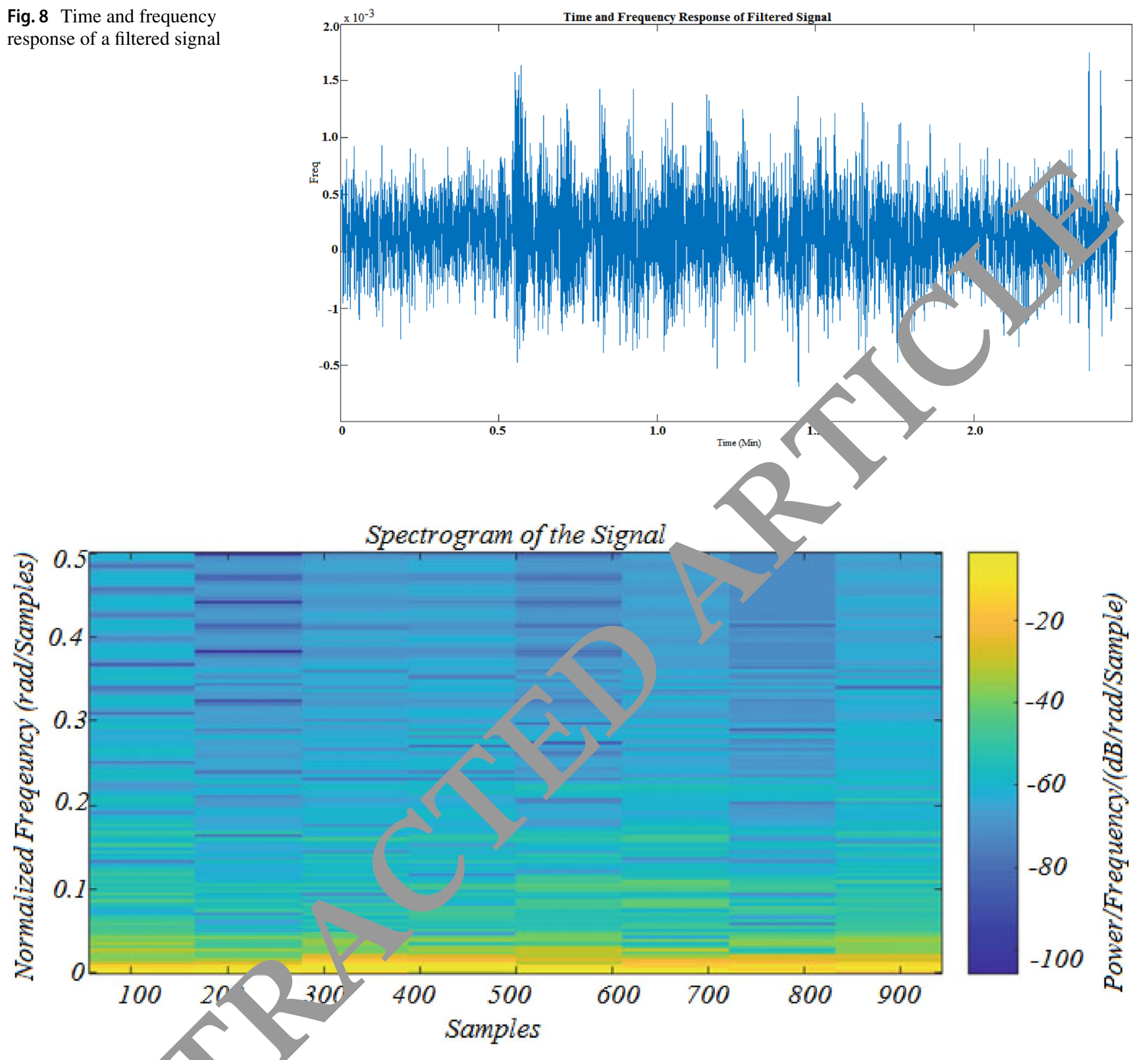

Fig. 9 Spectro am of a spe / signal

infect: The ve se transcription-polymerase chain reactio (RT .PCR testing strategy is used to determine the COV 19 virus. This RT-PCR processing is more expensive and mducing social distancing rules violation, and time-consuming. Therefore, this research work introduces the Generative Adversarial Network (GAN) based deep learning method to detect COVID-19 from speech signals quickly. As compared with existing methods, the proposed GAN method achieves a good result. The precision, recall, accuracy, and F-measure are $96.54 \%, 96.15 \%, 98.56 \%$, and 0.96 , respectively. 

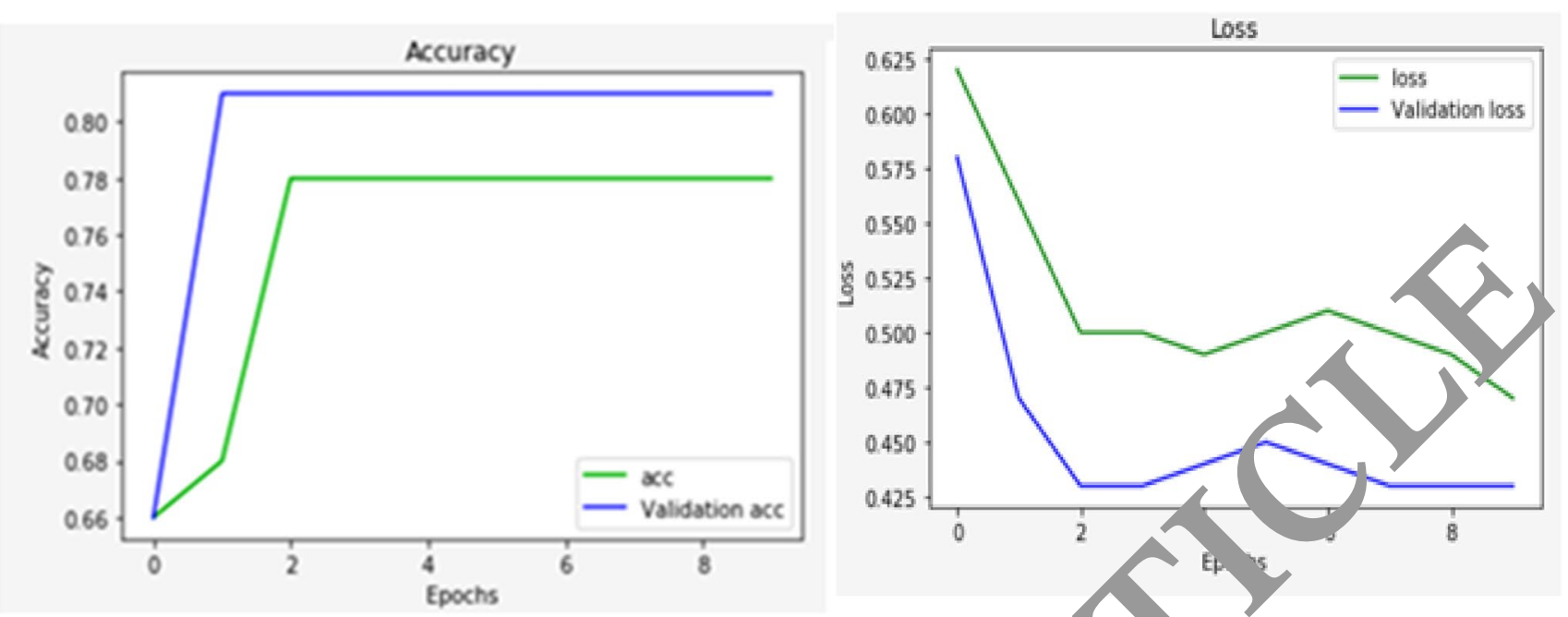

Fig. 10 Validation accuracy and loss during the training

Fig. 11 Performance analysis of classification ratio

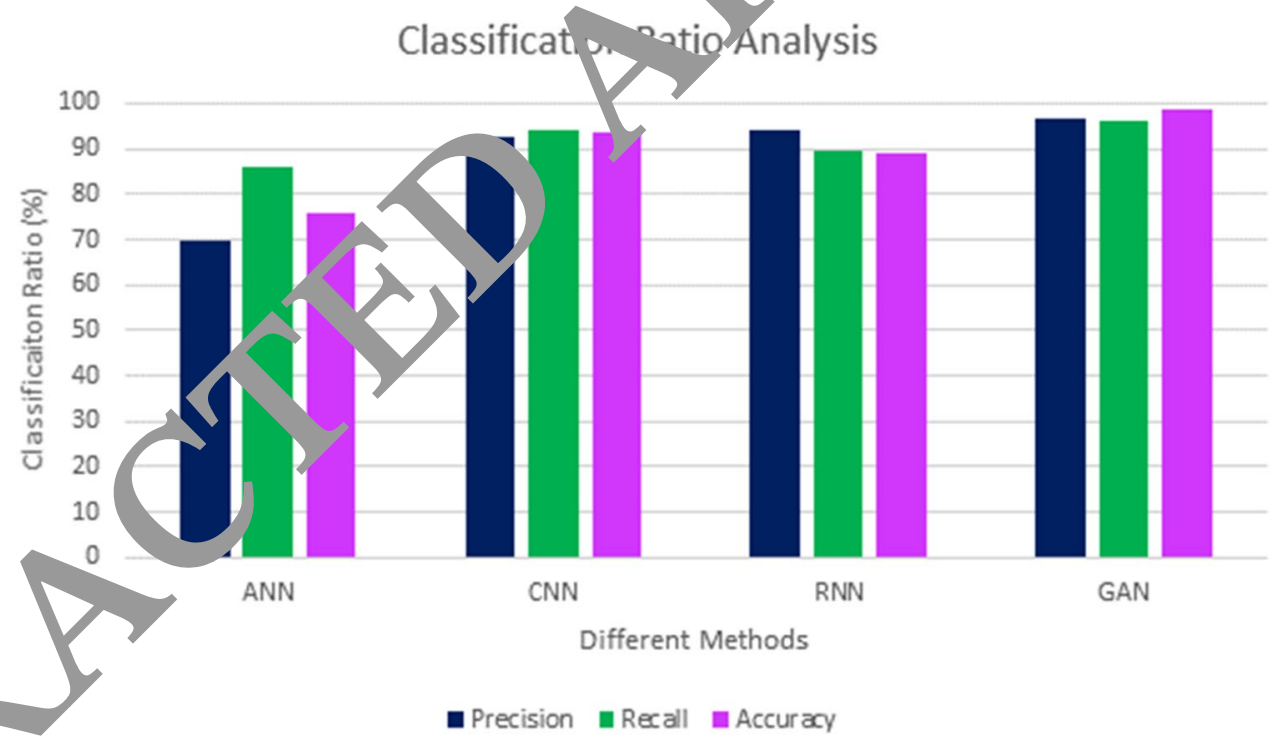

techniques. International Journal of Speech Technology, 23, 713-725. https://doi.org/10.1007/s10772-020-09712-z

Botha, G., Theron, G., Warren, R., Klopper, M., Dheda, K., Van Helden, P., \& Niesler, T. (2018). Detection of tuberculosis by automatic cough sound analysis. Physiological Measurement. https://doi.org/10.1088/1361-6579/aab6d0

Breathing sounds for COVID-19. Retrieved from May 8, 2020, from https://breatheforscience.com/

Chon, Y., Lane, N. D., Li, F., Cha, H., \& Zhao, F. (2012). Automatically characterizing places with opportunistic crowdsensing using smartphones. In: Proceedings of the ACM Conference on Ubiquitous Computing (UbiComp). Pittsburgh, PA, pp. 481-490.

Ghosh, S., Laksana, E., Morency, L.-P., \& Scherer, S. (2015). Learning representations of effect from speech. CoRR, vol. abs/1511.04747.

Ghosh, S., Laksana, E., Morency, L.-P., \& Scherer, S. (2016a). Representation learning for speech emotion recognition. In: Proceedings of Interspeech 2016. 
Ghosh, S., Laksana, E., Morency, L.-P., \& Scherer, S. (2016b). An unsupervised approach to glottalin verse filtering. In: Proceedings of EUSIPCO 2016.

Huber, J. E., \& Stathopoulos, E. T. (2015). Speech Breathing Across the Life Span and in Disease, Ch. 2 (pp. 11-33). Wiley.

Indian Institute of science-Coswara: A sound-based diagnostic tool for covid19. Retrieved from May 8, 2020, from https://coswa ra.iisc.ac.in/

James, A. P. (2015). Heart rate monitoring using human speech spectral features. Human-Centric Computing and Information Sciences, 5(1), 1-12.

McKeown, G., Valstar, M., Cowie, R., Pantic, M., \& Schroder, M. (2012). The Semaine database: Annotated multimodal records of emotionally colored conversations between a person and a limited agent. IEEE Transactions on Affective Computing, 3(1), 5-17.

Menni, C., Valdes, A. M., Freidin, M. B., Sudre, C. H., Nguyen, L. H., Drew, D. A., Ganesh, S., Varsavsky, T., Cardoso, M. J., El-Sayed Moustafa, J. S., Visconti, A., Hysi, P., Bowyer, R. C. E., Mangino, M., Falchi, M., Wolf, J., Ourselin, S., Chan, A. T., Steves, C. J., \& Spector, T. D. (2020). Real-time tracking of self-reported symptoms to predict potential COVID-19. Nature Medicine. https://doi. org/10.1038/s41591-020-0916-2

Nandakumar, R., Gollakota, S., \& Watson, N. (2015). Contactless sleep apnea detection on smartphones. In: Proceedings of the 13th Annual International Conference on Mobile Systems, Applications, and Services (MobiSys). Florence, Italy, pp. 45-57.

Oletic, D, \& Bilas, V. (2016). Energy-efficient respiratory sounds are sensing for personal mobile asthma monitoring. IEEE Sensors Journal, 16(23), 8295-8303.

Porter, P., Abeyratne, U., Swarnkar, V., Tan, J., Ng, T.-W., Brisbane, J. M., Speldewinde, D., Choveaux, J., Sharan, R., Kosasih, K., e dr. (2019). A prospective multicentrestudy was testing the dia ostic accuracy of an automated cough sound centered analytic sy. n for the identification of common respiratory disorder in childre. Respiratory Research, 20(1), 81.

Pramono, R. X. A., Bowyer, S., \& Rodriguez-Vi 'egas, E. 017). Automatic adventitious respiratory sound a alysis: A syst matic review. PLOS ONE. https://doi.org/10.1371 ournal.po le.0177926

Praveen Sundar, P. V., Ranjith, D., Karthikey T., e al. (2020). Low power area efficient adapti FIR filter hearing aids using distributed arithmetic archite ctu. nornational Journal of Speech Technology, 23, 287-296. https://doi.org/10.1007/ s10772-020-09686-y

Rachuri, K. K., Musolesi, M., Mascolo, C., Rentfrow. P. J., Longworth, C., \& Aucinas, A. (2010). EmotionSense: A mobile phones-based adaptive platform for experimental social psychology research. In: Proceedings of the ACM Conference on Ubiquitous Computing (UbiComp). Copenhagen, Denmark, pp. 281-290.

Sakai, M. (2015). Modeling the relationship between he $\rightarrow$ sate and features of vocal frequency. International Journa' of Co ' nputer Applications, 120(6), 32-37.

Schuller, B., Friedmann, F., Eyben, F. (2014). The Munich is oice Corpus: effects of physical exercising, h rate an s s conductance on human speech production. 1 : Pro eding of the 9th International Conference on Langua, e Resources avaluation, Reykjavik, Iceland, 26-31 May 20 4, pp. 1505-1510.

Thorpe, W., Kurver, M., King, G \& i ome, ${ }^{\prime}$. (2001). Acoustic analysis of cough. In: Proceea of of in weventh Australian and New Zealand Intelligent informa . Systems Conference. IEEE, pp. 391-394.

Trouvain, J., \& Truong, K P. ( 4 5). Prosodic characteristics of reading speech bef nd after $\mathrm{t}$ admill running. In: 16th Annual Conference the I ternational Speech Communication Association, Dresden, mang, september 6-10, 2015.

Usman, M. (2017). On performance degradation of speaker recognition due to variation in speech characteristics caused by physiol gi ar anges. International Journal of Computing and Digital s stems (IJCDS), 6(3), 119-126.

-ata Srika in, N., \& Strik, H. (2019). Deep sensing of breathing nal during conversational speech.

Windm n, A., Minakshi, M., Bharti, P., Chellappan, S., Johansson, N., Jenkins, B. A., \& Athilingam, P. R. (2018). Tussiswatch: A smartphone system to identify cough episodes as early symptoms of chronic obstructive pulmonary disease and congestive heart failure. IEEE Journal of Biomedical and Health Informatics, 23(4), 1566-1573.

Publisher's Note Springer Nature remains neutral with regard to jurisdictional claims in published maps and institutional affiliations. 